\title{
Nonstandard electroconvection in a bent-core oxadiazole material
}

\author{
S. Kaur, ${ }^{1}$ A. Belaissaoui, ${ }^{2}$ J. W. Goodby, ${ }^{2}$ V. Görtz, ${ }^{2}$ and H. F. Gleeson ${ }^{1}$ \\ ${ }^{1}$ School of Physics and Astronomy, University of Manchester, Manchester M13 9PL, United Kingdom \\ ${ }^{2}$ Department of Chemistry, University of York, York YO10 5DD, United Kingdom
}

(Received 9 November 2010; revised manuscript received 20 January 2011; published 8 April 2011)

\begin{abstract}
Electroconvection (EC) phenomena have been investigated in the nematic phase of a bent-core oxadiazole material with negative dielectric anisotropy and a frequency dependent conductivity anisotropy. The formation of longitudinal roll (LR) patterns is one of the predominant features observed in the complete frequency and voltage range studied. At voltages much above the LR threshold, various complex patterns such as the "crisscrossed" pattern, bimodal varicose, and turbulence are observed. Unusually, the nonstandard EC (ns-EC) instability in this material, is observed in a regime in which we measure the dielectric and conductivity anisotropies to be negative and positive respectively. A further significant observation is that the EC displays distinct features in the high and low temperature regimes of the nematic phase, supporting an earlier report that EC patterns could distinguish between regions that have been reported as uniaxial and biaxial nematic phases.
\end{abstract}

DOI: 10.1103/PhysRevE.83.041704

PACS number(s): 61.30.-v, 47.54.-r, 47.20.Lz

\section{INTRODUCTION}

Nonequilibrium systems form interesting patterns, driven either by temperature through buoyancy forces (RayleighBenard convection) or electric field through space charges (electrohydrodynamic convection) [1]. Studying electrohydrodynamic convection offers an advantage for the study of complex anisotropic systems, with an opportunity to understand the unusual nonlinear couplings and patterns based on instability mechanisms, which are not available in isotropic systems. In comparison to isotropic systems, liquid crystalline phases are excellent candidates for studying pattern formation mechanisms as the anisotropy in their physical properties can be changed with temperature.

Nematic liquid crystals, being the simplest liquid crystalline phase, have been extensively studied for the vast display of patterns that can occur on the application of an electric field [2-4]. Pattern formation is mainly influenced by the geometry (planar or homeotropic) and anisotropy of the system. One can define the dielectric anisotropy, $\Delta \varepsilon$, as $\varepsilon_{\|}-\varepsilon_{\perp}$ and the electrical conductivity anisotropy, $\Delta \sigma$, as $\sigma_{\|}-\sigma_{\perp}$ where $\varepsilon_{\|}\left(\sigma_{\|}\right)$and $\varepsilon_{\perp}\left(\sigma_{\perp}\right)$ are the dielectric (conductivity) components measured parallel and perpendicular with respect to the nematic director $\boldsymbol{n}$, respectively. Various sign combinations of the dielectric and conductivity anisotropy give rise to different electroconvection (EC) patterns. The earliest model developed to describe some of the observed instability processes was the Carr-Helfrich model (later also called the standard model for three-dimensional cases), which predicted the possibility of EC in the $(-,+)$ case, where the first sign corresponds to dielectric anisotropy and the second to conductivity anisotropy [5-7]. A similar convention will be used for the rest of the paper. In the most conventional $(-,+)$ case described by Carr-Helfrich theory, above a threshold voltage $U_{\text {th }}$ one observes a pattern of convective rolls either perpendicular or oblique with respect to the director $\boldsymbol{n}$ with the wavelength of the rolls, $\lambda$, about the cell thickness $d$. This is the low frequency conductive regime. Above a certain cutoff frequency $f_{c}$, the regime is known as dielectric, in which the wavelength of the rolls is only a few $\mu \mathrm{m}(3-4 \mu \mathrm{m}$ in the case of a standard nematic material such as $\mathrm{N}$-(4-methoxybenzylidene)4-butylaniline (MBBA) [2]), is independent of $d$, and is a function of a combination of material parameters. The wavelength of the dielectric rolls for typical cells $(d>10 \mu \mathrm{m})$ is much smaller than in the conductive regime [2]. Other cases, such as $(+,-)[8-10],(+,+)[11,12]$, and $(-,-)[13-17]$, have been studied and novel patterns observed. For the $(+,+)$ case, the material can develop Carr-Helfrich rolls, provided that the Freederickz threshold is above the frequency dependent threshold for EC $[2,11]$. The $(-,-)$ case has shown EC that is not described by the Carr-Helfrich model and the patterns observed are categorized as nonstandard EC (ns-EC). It needs to be mentioned here that the EC pattern depends not just on the sign but also on the magnitude of anisotropies [2,13]. ns-EC was first reported more than three decades ago [18,19], and more recently by Kochowska et al. [13] in nematic liquid crystals. The characteristic features were analogous to the standard model but the origin of these patterns could not be explained by the classical Carr-Helfrich model. For instance, the observance of dark and bright stripes parallel to the director is in contrast to the perpendicular stripes observed in the case of the standard model. The low contrast, dynamic behavior at the onset of parallel stripes or rolls, or the threshold being a linear function of frequency and thickness of the sample cell are few of the features that distinguish ns-EC from standard EC. Various scientists have explained the unusual dynamics in a number of ways: a qualitative argument based on "destabilization of twist fluctuations" [18], a speculation on an isotropic mechanism (based on the nonuniform space charge distribution along the field) [19], and the flexoelectric effect [20-22]. Another significant pattern is the prewavy instability [23,24], which cannot be described by the standard Carr-Helfrich model $[2,25]$. The pattern is best observed between crossed polarizers and is a series of bright and dark stripes perpendicular to director orientation with a wavelength much larger than the thickness of the cell $(\lambda \approx 4 d-8 d)$. The mechanism producing this instability is not well understood but it has characteristics which have some similarities to better understood models, such as the anisotropic inertia mode and the isotropic electrolyte mode [14]. 
For the present investigation, a bent-core oxadiazole nematic material has been studied that exhibits unusual electroconvection patterns. The inspirations for researchers to study bent-core materials include the discovery of spontaneous polar order in their smectic phases [26] and reports of biaxial nematic phases in some materials $[27,28]$, though we note that the occurrence of biaxiality in thermotropic nematic materials remains contentious [29]. The oxadiazoles are promisingly strong contenders for nematic biaxiality and possible ferroelectric nematic switching, though they suffer from high transition temperatures. The material considered here has been reported to have both uniaxial and biaxial nematic phases, characterized via polarized Raman spectroscopy and x-ray scattering [30]. Despite the fact that there is still a debate about biaxiality in oxadiazole materials, the EC phenomenon detailed in this paper nonetheless characterizes the interesting distinction between the high and low temperature regions of the nematic phase, highlighting either a transition from the uniaxial to the biaxial phase or, if recent reports are found to be a better description of these systems, a lower temperature cybotactic regime [29]. Irrespective of this ongoing debate, further study of the electro-optic properties of materials reported as biaxial will contribute to a better understanding of them.

Although a lot of work has been reported on EC phenomena in various bent-core materials $[9,10,12,14,16,17]$, there are no detailed reports on such behavior in the oxadiazole systems. Xiang et al. reported a preliminary observation of EC patterns in an oxadiazole material [31]. This paper now presents a detailed study of the characteristic EC patterns exhibited by the material.

The paper is structured as follows. It begins with details of the experimental setup, the material, and the methods employed. A detailed comparative study of the patterns observed in fresh and old sample cells is presented to highlight the changes that can occur as a result of the high working temperature in the bent-core material. This is followed by a discussion of the material's dielectric and conductivity anisotropy. The paper is then broadly segregated into two sections. The first part introduces the different EC patterns that form at various frequencies and voltages and analyzes the formation of the rolls. This is followed by a study of the behavior of the patterns with temperature in the nematic phase. The second part of the paper shows that we observe significant differences in the EC patterns in the temperature regimes that have been reported as biaxial and uniaxial nematic phases. Specifically, changes in the longitudinal rolls and the bimodal varicose patterns in the two regimes are explicitly shown. Last, we compare and contrast the ns-EC pattern formation in this bent-core material with other reports.

\section{EXPERIMENT}

The bent-core material under study is $\mathrm{C} 5-\mathrm{Ph}-\mathrm{ODBP}-\mathrm{Ph}$ OC12, shown in Fig. 1, which has the phase transition sequence

Isotropic $215^{\circ} \mathrm{C} N_{u} 188-183^{\circ} \mathrm{C} N_{b} 160^{\circ} \mathrm{C} \mathrm{Sm}-X$.

$N_{u}$ and $N_{b}$ denote uniaxial and biaxial nematic phases respectively, and $\mathrm{Sm}-X$ is an unidentified smectic phase. The range of temperatures that cover the $N_{u}$ to $N_{b}$ phase indicates

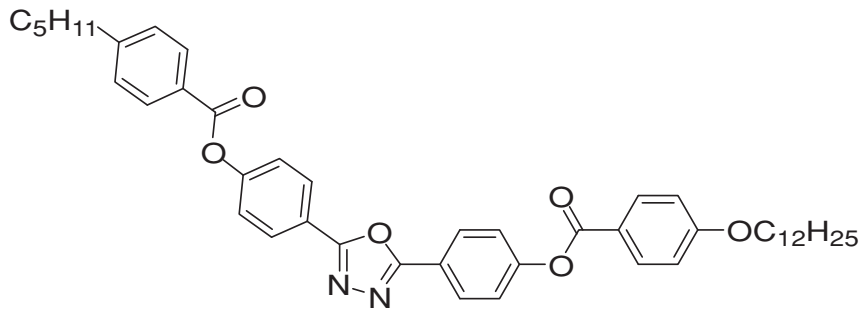

FIG. 1. Molecular structure of the bent-core material $\mathrm{C} 5-\mathrm{Ph}$ ODBP-Ph-OC12.

the ambiguity of determining a second order uniaxial to biaxial transition in this system [30].

For the electroconvection measurements, $\sim 5-\mu$ m-thick sandwich cells (AWAT PPW, Poland) with an active electrode area of $30 \mathrm{~mm}^{2}$ were used. Due to the high temperature regime of the nematic phases, a high temperature polyimide alignment layer (SE130) was used to induce planar-boundary conditions and a high temperature conducting glue (Silver Adhesive 503, $62 \%$ weight solids, Electron Microscopy Sciences) was used to attach the wires to the electrode surfaces. The devices were capillary filled in the nematic phase at $190^{\circ} \mathrm{C}$. The electro-optic setup included a Leica DM 2500P polarizing microscope equipped with a Linkam TMS 93 temperature controller and a THMS 600 hot stage with relative temperature control accurate to $\pm 0.1^{\circ} \mathrm{C}$. The alternating voltage across the cell was applied using an Agilent 33220A, 20-MHz function arbitrary wave form generator, amplified via an in-house built amplifier. An Agilent 34401 A digital multimeter was used to read the applied voltage. Transmitted light intensity measurements were carried out with the sample held between crossed polarizers on a polarizing microscope, using white light and a photodiode to detect the light. The use of a $20 \times$ objective limited the data collection area to $\mathrm{a} \sim 1$-mm-diameter region close to the center of the electrode area. The measurements were normalized with respect to the recorded optical transmission through an empty cell with the top polarizer removed. A Delta Pix DP200 camera with a sensor resolution of $1024 \times 768$ pixels was used to record the images. ImageJ $1.43 \mathrm{u}$ software was used for the analysis of the EC patterns obtained. The images taken were uploaded in the software and the thickness of the stripes was measured using the various tools available.

As mentioned above, the bent-core oxadiazole liquid crystal under study is a high temperature material (clearing point $>200^{\circ} \mathrm{C}$ ) and most of the standard aligning agents soften above $160^{\circ} \mathrm{C}$. Our earlier preliminary EC studies [31] were carried out on this material using standard polyimide as the aligning agents. However, we observed that a freshly prepared cell changes within a few hours of filling. The dielectric permittivity and conductivity, which are significant parameters in the formation of the EC patterns, change by a factor of 10 over a few hours. Xiang et al. reported on the observation of two EC instabilities: prewavy 1 and prewavy 2 [31]. According to the paper, prewavy 1 existed in the uniaxial phase with stripes parallel to the rubbing direction and a periodicity of the order of cell thickness. On the other hand, the prewavy 2 instability has stripes perpendicular to the rubbing direction and with a similar periodicity as prewavy 1 , and existed in the entire nematic regime. We observed that these patterns 

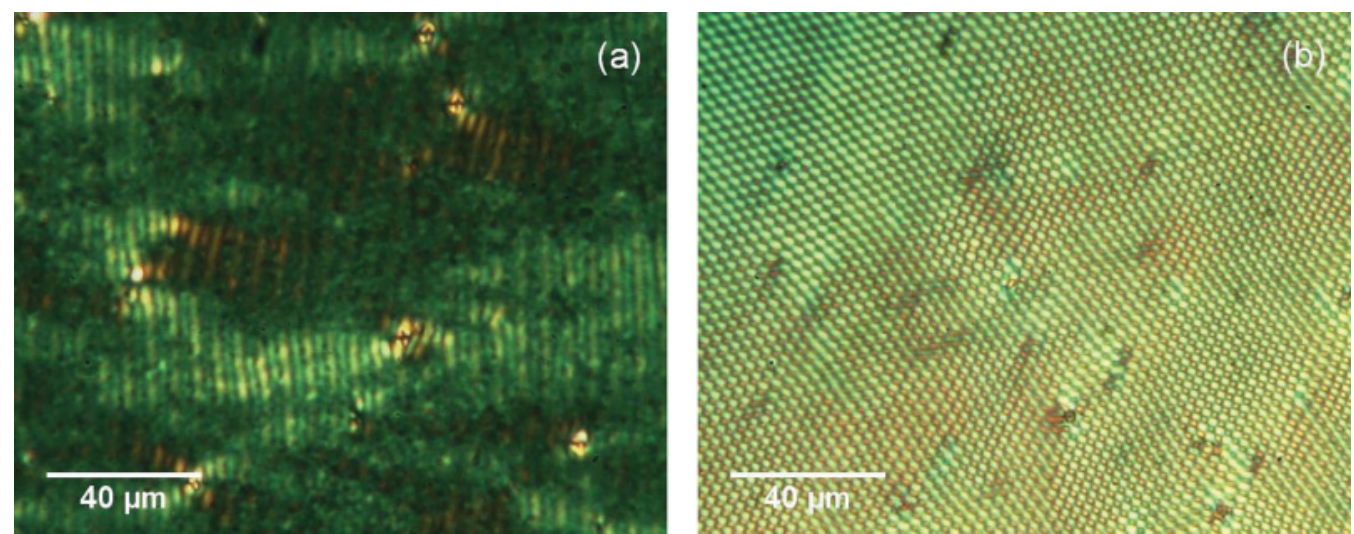

FIG. 2. (Color online) EC pattern in a sample cell with standard polyimide as the alignment layer, showing the change in pattern a day after filling. EC pattern observed at $T-T_{\mathrm{NI}}=-20 \mathrm{~K}$ in (a) a freshly prepared cell at $1.21 U_{\text {th }}$ where $U_{\text {th }}=9.05 V_{\text {rms }}$, (b) one day after filling at $1.12 U_{\text {th }}$ where $U_{\text {th }}=5.63 V_{\text {rms. }}$. The (crossed) polarizer and analyzer are at $\sim 45^{\circ}$ to the rubbing direction which is in vertical direction in both (a) and (b). The scale is in the lower left hand corner in each picture.

are exhibited when the cells have been kept for a few hours to a number of days, an aging phenomenon which was also reported by Wiant et al. [14] in another bent-core material. Measurements have revealed that the pattern of longitudinal rolls seen in a fresh cell changed to a beadlike pattern the following day. Figure 2 distinguishes between the two types of EC patterns seen in a device which used a standard polyimide for alignment. Figure 2(a) shows the longitudinal rolls as observed in a freshly prepared cell, which changed into a beady pattern the next day, Fig. 2(b). Both photomicrographs were taken at the same reduced temperature (temperature below the nematic to isotropic transition) of $T-T_{\mathrm{NI}}=-20 \mathrm{~K}$ and at a frequency of $1 \mathrm{kHz}$. The threshold voltage for the EC also decreased and one observes the pattern at a lower value of voltage even for identical conditions of temperature and frequency.

Taking into account the importance of using fresh cells and to understand the true mechanism of EC patterns in these high temperature materials, a different alignment layer (SE130 from Nissan Chemicals) was used. After preliminary measurements it was clear that even with the high temperature alignment layer the EC pattern changed after 8-10 h. A complete set of measurements takes 5-6 h after filling, thus leaving sufficient time between the last measurement and any significant change in the cell. Hence all the measurements reported in the following sections have been carried out on freshly filled cells. For the anisotropy measurements, good homeotropic alignment of the material was required. Most standard homeotropic alignment agents failed to align this bent-core material, though we found that good alignment across the temperature range could be obtained using trichloro octa decyl silane in heptane.

\section{ANISOTROPIC PROPERTIES}

As mentioned in the Introduction, one of the deciding factors for the type of EC pattern is the combination of signs as well as the magnitude of the dielectric and conductivity anisotropy. One can clearly observe a Freederickz transition (at fields below $\sim 0.5 \mathrm{~V} / \mu \mathrm{m}$ ) in the homeotropic cell, Fig. 3(a). Insets in the figure show the change from a homeotropic to a quasihomogeneous texture at low and high electric fields, respectively. The dielectric permittivity $\left(\varepsilon^{\prime}\right)$ and conductivity $(\sigma)$ values have been obtained in the homeotropic geometry via application of an electric field, shown in Figs. 3(b) and 3 (c), at a frequency of $1 \mathrm{kHz}$ for different temperatures across the nematic regime. Below the Freederickz transition the homeotropic configuration gives the $\varepsilon_{\|}\left(\sigma_{\|}\right)$component and at sufficiently high electric fields the quasihomogenous configuration attained allows us to estimate the perpendicular components, $\varepsilon_{\perp}\left(\sigma_{\perp}\right)$. A small increase in $\varepsilon^{\prime}$ [Fig. 3(b)] is seen below the point of the Freedericksz threshold observed optically [Fig. 3(a)]. Further, the dielectric data saturate at around $1 \mathrm{~V} / \mu \mathrm{m}$ whereas the optical data continue to change. This clearly complex behavior merits further study, and we suggest that these qualitative differences in behavior are due to the influence that the high conductivity of the sample has on the dielectric measurements. Although the dielectric constant saturates at the higher fields applied, the conductivity does not. The important factor for the EC studies is that it is clear from our data that the material under study has negative dielectric and positive conductivity anisotropy at 1 $\mathrm{kHz}$, though the absolute values have not been determined. There is no sign inversion of dielectric anisotropy with temperature or frequency. On the other hand, an inversion is seen at all temperatures from $+\Delta \sigma$ (at lower frequencies) to $-\Delta \sigma$ at high frequencies (well above those at which we report observation of EC in this paper); see inset to Fig. 3(c) for $T-T_{\mathrm{NI}}=-36 \mathrm{~K}$. This factor is important in discussing the EC results that we present in the following sections.

\section{PATTERN FORMATION}

The occurrence and types of EC behavior have been studied over the frequency range from $20 \mathrm{~Hz}$ to $2 \mathrm{MHz}$ across the entire $55 \mathrm{~K}$ wide nematic regime. On application of a voltage, the first observance of an EC pattern under the polarizing microscope shows alternate bright and dark stripes of wavelength equal to nearly twice the thickness of the cell under crossed (or nearly crossed) polarizers. Though these stripes appear to be similar to standard EC (s-EC), detailed examination shows that they 

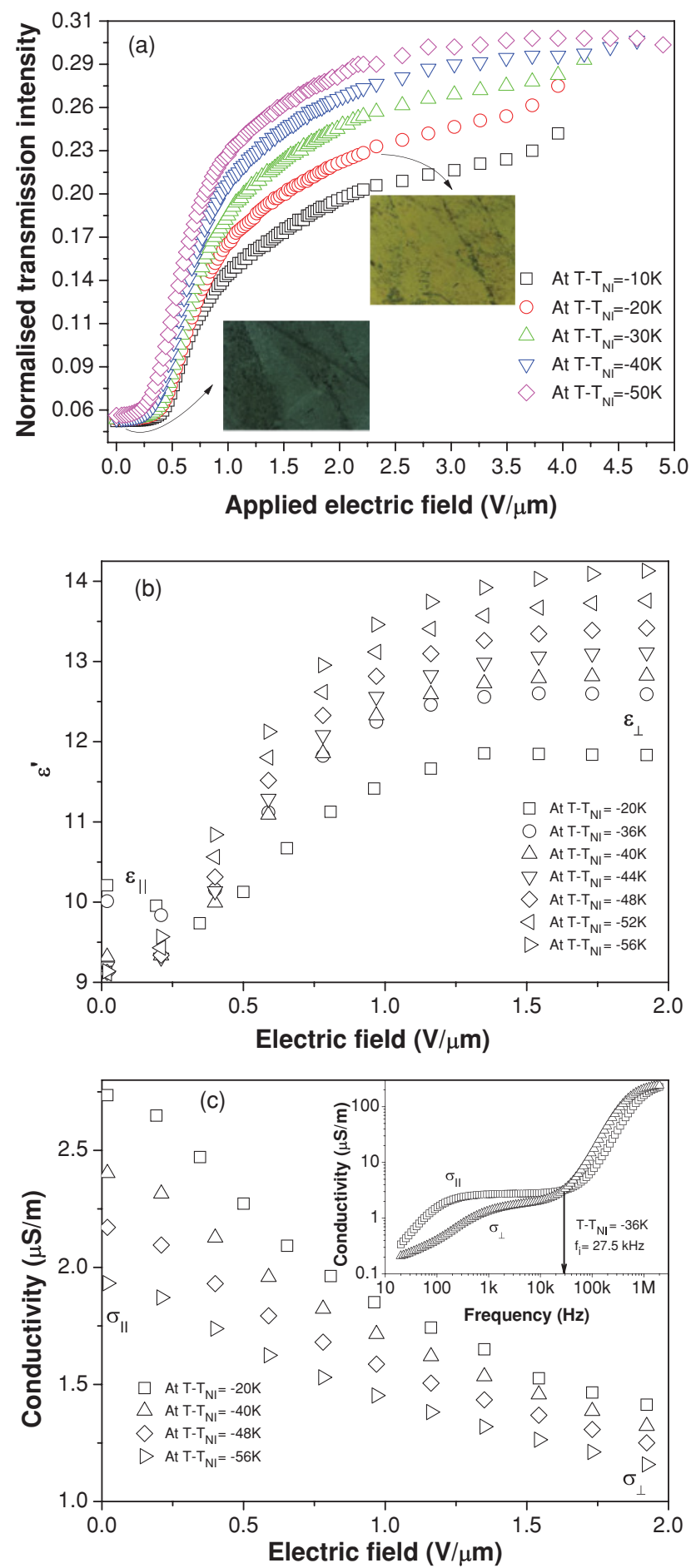

FIG. 3. (Color online) (a) Normalized transmission intensity as a function of applied electric field $(1 \mathrm{kHz})$ in a $2.6-\mu$ m-thick homeotropic cell. Insets show the textures in both homeotropic and quasi-homogeneous configuration. (b) Parallel $\left(\varepsilon_{\|}\right)$and perpendicular $\left(\varepsilon_{\perp}\right)$ dielectric components, and (c) parallel $\left(\sigma_{\|}\right)$and perpendicular $\left(\sigma_{\perp}\right)$ conductivity values as a function of electric field $(1 \mathrm{kHz})$ at different reduced temperatures. Inset in (c) shows the conductivity anisotropy change with frequency inversion at $f_{i}=27.5 \mathrm{kHz}$ for $T-T_{\mathrm{NI}}=-35 \mathrm{~K}$.

are not and hence we categorize them as ns-EC. The first distinct feature is the weak contrast of the patterns at the onset as opposed to that observed in s-EC. The patterns cannot be observed with a single polarizer and always require crossed or nearly crossed polarizers to observe their presence. Hence one cannot produce a shadowgraph image near the onset of these ns-EC patterns. Another important point of distinction is the orientation of the ns-EC, which is along the molecular director $\boldsymbol{n}$, unlike s-EC rolls which run perpendicular to $\boldsymbol{n}$. Due to their orientation, the term longitudinal rolls (LRs) or parallel rolls (PRs) is given to such EC patterns. A clear distinction of the characteristic features of LRs and PRs is not given in the literature. As the rolls observed in our case are not exactly parallel to $\boldsymbol{n}$, we use the terminology longitudinal rolls.

Figure 4 shows frequency dependence of the voltage thresholds of various EC patterns observed at a temperature of $T=T_{\mathrm{NI}}-13 \mathrm{~K}\left(T_{\mathrm{NI}}=215^{\circ} \mathrm{C}\right.$ is the isotropic to nematic transition temperature) across the complete frequency range. Just above the threshold for EC near $4 \mathrm{~V}$, there is an appearance of a "faint crisscrossed" pattern, followed by traveling oblique rolls (ORs) at higher voltages changing to LR depending on the frequency. Near $11 \mathrm{~V}$ and above, at $\sim 900 \mathrm{~Hz}$, a wavy pattern perpendicular to the LR is observed which deforms on further increasing the frequency. On increasing the voltage above $14 \mathrm{~V}$, near $500 \mathrm{~Hz}$ and above, in addition to LRs, one observes patterns being formed in the direction perpendicular to the LR and hazy patterns traveling over the whole field of view. In Fig. 4 this region is marked by ovals and intersecting lines representing a number of EC patterns. At $16 \mathrm{~V}$ and above, at low frequencies, there is not a clear line of distinction between the ORs and LRs. At higher voltages and higher frequencies (2-4 kHz), the LRs become less visible and are quite faint. The EC patterns were investigated in the frequency range up to $2 \mathrm{MHz}$ but no EC pattern of any kind is observed above the frequency end region marked in the figure. Thus at $4 \mathrm{~V}$,

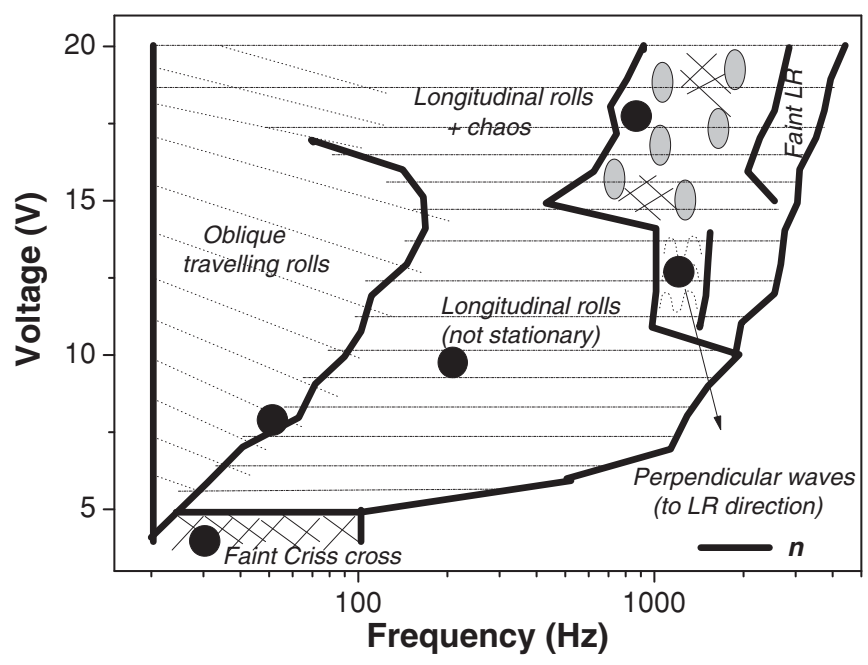

FIG. 4. Occurrence of EC patterns in the voltage and frequency regime at $T-T_{\mathrm{NI}}=-13 \mathrm{~K}$. The direction of rolls is denoted by the dotted lines with respect to the direction of the director $\boldsymbol{n}$. The lines showing transitions between the different types of EC patterns are an approximation only as there is an interpenetration of rolls in the regions as indicated. The bold circular dots represent the EC patterns that are explicitly shown in Fig. 5. 

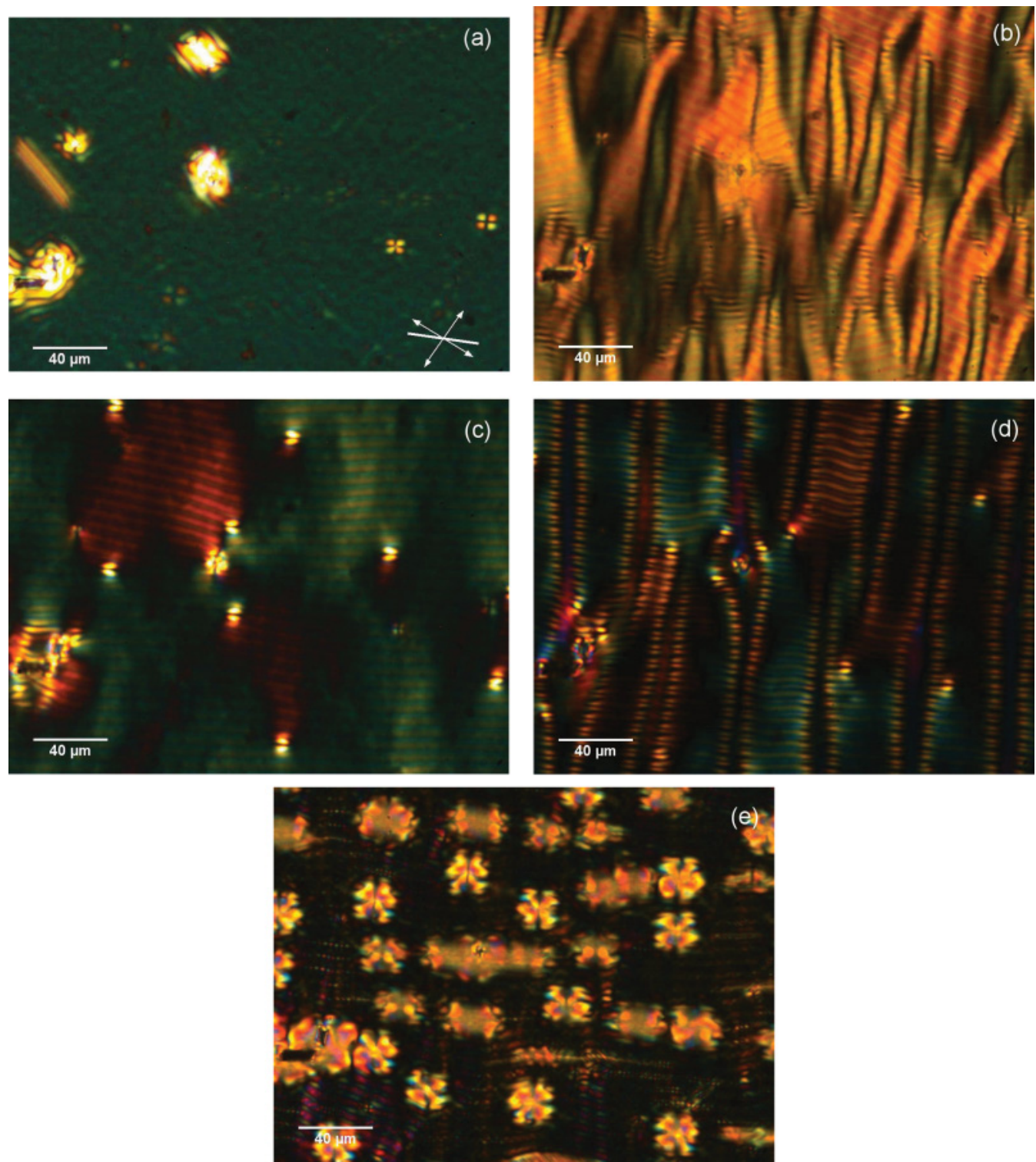

FIG. 5. (Color online) Typical EC patterns observed at $T-T_{\mathrm{NI}}=-13 \mathrm{~K}$ as marked by bold circular dots in Fig. 4 showing the appearance of (a) crisscross pattern at $4 V_{\text {rms }}$ and $20 \mathrm{~Hz}$, (b) oblique rolls at $8 V_{\mathrm{rms}}$ and $50 \mathrm{~Hz}$, (c) longitudinal rolls at $10 V_{\mathrm{rms}}$ and $220 \mathrm{~Hz}$, (d) wavy pattern + longitudinal rolls at $13 V_{\mathrm{rms}}$ and $1.1 \mathrm{kHz}$, and (e) higher instabilities along with longitudinal rolls at $18 V_{\mathrm{rms}}$ and $800 \mathrm{~Hz}$. The director $\boldsymbol{n}$ is along the roll direction, i.e., in the almost horizontal direction as marked by the solid line in the bottom right hand corner of (a). The double-headed arrows show the orientation of the crossed polarizers. The scale is shown at the lower left hand corner in each picture.

EC is seen up to frequencies of $100 \mathrm{~Hz}$ and this maximum frequency increases to $4.3 \mathrm{kHz}$ at $20 \mathrm{~V}$. The bold dark lines which segregate different regions are the best obtained values for the changing EC pattern at different voltages and frequency.

Figure 5 shows typical EC patterns representing different regions as marked by the bold black dots in Fig. 4. At the start of EC with an applied voltage of $4 \mathrm{~V}$ and frequency $20 \mathrm{~Hz}$, one could just observe the faint crisscrossed pattern shown in Fig. 5(a), which transforms to oblique traveling rolls and LR at higher voltages and frequencies. Apart from the oblique traveling roll region as shown in Fig. 5(b), LRs are seen at all frequencies and voltages either alone or accompanied by other
EC patterns, as seen in Figs. 5(c)-5(e). Unlike the transition from LR to normal rolls, observed in other materials [10,15], we observe only a transition from the oblique to longitudinal rolls, which remains for the complete frequency and voltage range studied in the present material. Figure 5(d) shows a wavy structure in the direction perpendicular to the LR. The approximate wavelength of the repeated wavy structure is eight times the thickness of the cell. Due to the spacer as well as dust particles, the structure appears broken and not uniform throughout. The wavelength of the structure corresponds to the prewavy characteristic wavelength as reported in Refs. [14] and [24]. According to Huh et al. the prewavy pattern is formed at voltages much higher than the Freederickz threshold and is 
so called due to its evolution into a sinusoidal wavy pattern with time [24, and references therein]. Another characteristic property is that it has best contrast when crossed polarizers are used, though it remains visible with parallel polarizer settings as well; removing one of the polarizers makes them undetectable [24]. In our case, at the threshold voltage of these wavy structures, within a few minutes the pattern evolves, looking similar to a wavy structure, as seen in Fig. 5(d), and the pattern has the best contrast with crossed or nearly crossed polarizers and is only very faintly observable with a single polarizer. However, despite these similarities, the prewavy structure as shown by Waint et al. and Huh et al. does not look similar to the one we observe [14,24].

In summary, unlike the standard EC observed in planar aligned $(-,+)$ nematic materials, the EC reported here is nonstandard, in the form of longitudinal and parallel rolls (LR and PR). Kochowska et al. [13] also reports ns-EC (parallel and oblique rolls: PR and OR) in materials with finite positive $\Delta \sigma$, while Tadapatri et al. [16] observed LR in materials extending well into the $(-,+)$ region. On the other hand, the occurrence of oblique rolls in the low frequency region could be either standard or nonstandard; the absence of a shadowgraph image at the onset is the best way to distinguish the cases [15]. In this work, the oblique rolls do not produce a shadowgraph image near the onset, confirming ns-EC.

It is also important to comment on the fact that the ns-EC observed in the form of LR or OR patterns is not stationary (it changes continuously with time). Figure 5(c) shows two types of domains: orange and green. These domains interchange positions so that the pattern displaces itself with time. Patterns at higher voltages, too, are displaced with time. The dynamics, studied by inclusion and tracking of particles in the system will be considered in a future publication.

Figure 6(a) shows the wavelength of the rolls as a function of frequency at different applied voltages at the same temperature of $T=T_{\mathrm{NI}}-13 \mathrm{~K}$. The complete regime can be segregated into two broad regions: one with oblique traveling rolls and other with longitudinal rolls. At low frequencies (up to $\sim 100 \mathrm{~Hz}$ ), the wavelength of the (oblique) rolls decreases with increasing frequency. A region then follows where the wavelength of the longitudinal rolls is nearly independent of the frequency of the applied voltage (within an uncertainty of $\pm 5 \%$ ). At even higher frequencies the wavelength of the LRs becomes frequency dependent; the threshold to this phenomenon is voltage dependent. The bold blue vertical line near $300 \mathrm{~Hz}$ marks the beginning of the second frequency dependent regime for a voltage of $9 \mathrm{~V}$ (just one voltage is shown for simplicity). The behavior of rolls as a function of applied voltage is shown explicitly for two frequencies (200 Hz and $1 \mathrm{kHz}$ ) in Fig. 6(b). At $200 \mathrm{~Hz}$, below $14 \mathrm{~V}$, the roll wavelength is independent of the applied voltage but above that it becomes weakly voltage dependent. On the other hand at $1 \mathrm{kHz}$, below $14 \mathrm{~V}$, the roll wavelength is strongly voltage dependent, becoming weakly voltage dependent (similar to the low frequency behavior) above $14 \mathrm{~V}$. According to Penz [31], in a sample of fixed thickness and at a constant frequency, the spatial periodicity of the rolls is independent of voltage above the threshold voltage (in that case $3 \mathrm{~V}$ ). Tadapatri et al. reported on EC in bent-core nematic materials and observed both longitudinal and normal rolls in which the wavelength of
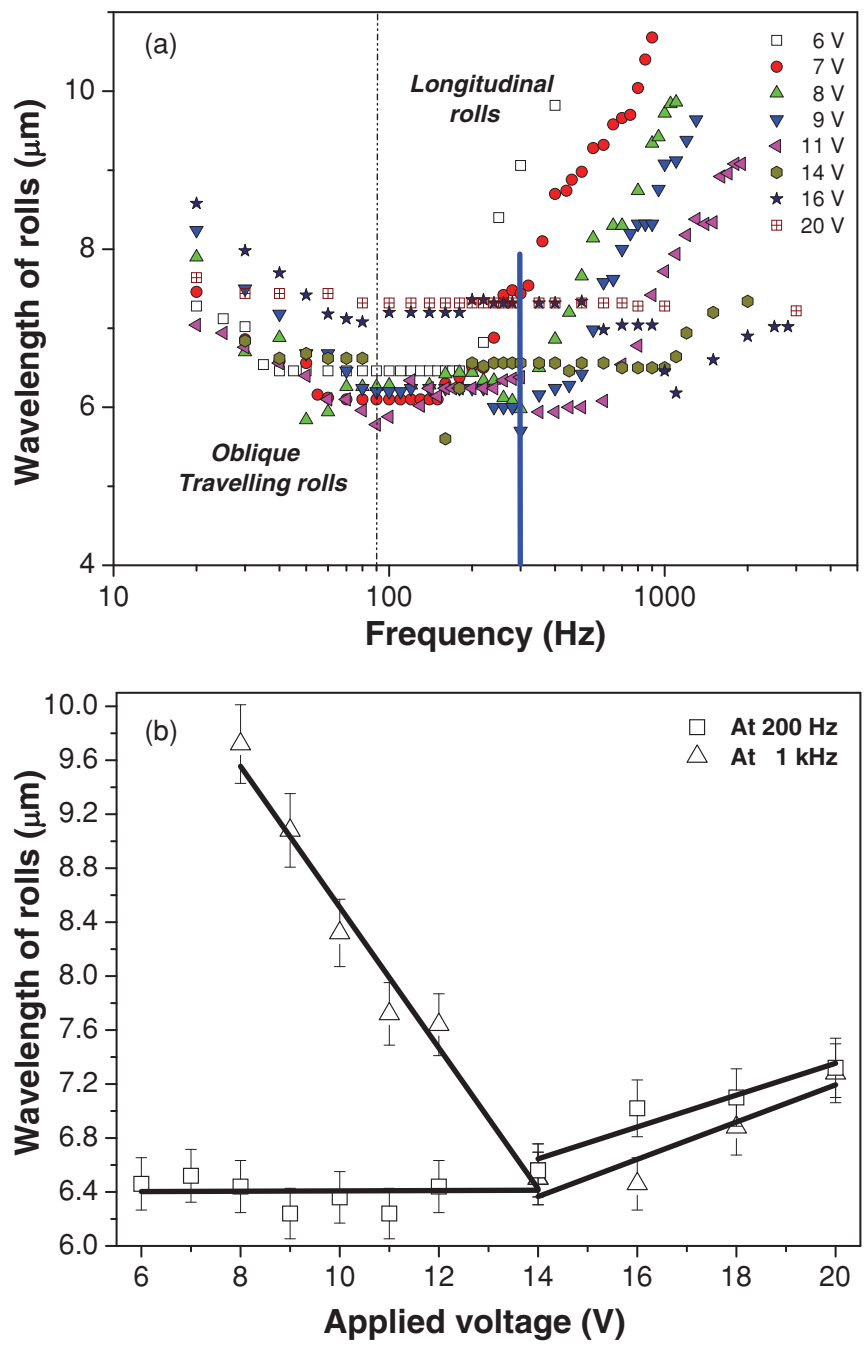

FIG. 6. (Color online) The wavelength of the oblique and longitudinal rolls at $T-T_{\mathrm{NI}}=-13 \mathrm{~K}$ (a) as a function of frequency at different voltages and (b) as a function of applied voltage at 200 $\mathrm{Hz}$ (open squares) and $1 \mathrm{kHz}$ (closed triangles). The dotted vertical line near $90 \mathrm{~Hz}$ in (a) shows the transition from oblique traveling rolls to longitudinal rolls. The vertical solid line near $300 \mathrm{~Hz}$ shows a typical transition to the regime where the roll wavelength increases as a function of frequency for one voltage $(9 \mathrm{~V})$. The uncertainty in measuring the roll wavelength is $5 \%$; the fitted lines are a guide to the eye.

the LR was found to be an increasing function of frequency in the range $10-100 \mathrm{kHz}$ [16]. However, in our case one sees a voltage independent roll wavelength only for conditions of low frequency and voltage. At higher frequencies there is no voltage regime for which the rolls have constant thickness. On the other hand, the threshold wavelength of the rolls in our case is $d<\lambda \leqslant 2 d$ for almost all of the frequency range, while it is typically $2 d-3 d$ in other works. The wavelength of rolls [Fig. 6(a)] is twice the thickness of the cell at low voltages $(<8 \mathrm{~V})$ and high frequencies. The threshold wavelength of the rolls (which is independent of voltage according to Penz [32]) is $6-6.6 \mu \mathrm{m}$, a little more than the cell thickness of $\sim 5 \mu \mathrm{m}$. In the whole frequency range studied, no transition to normal rolls is observed. 


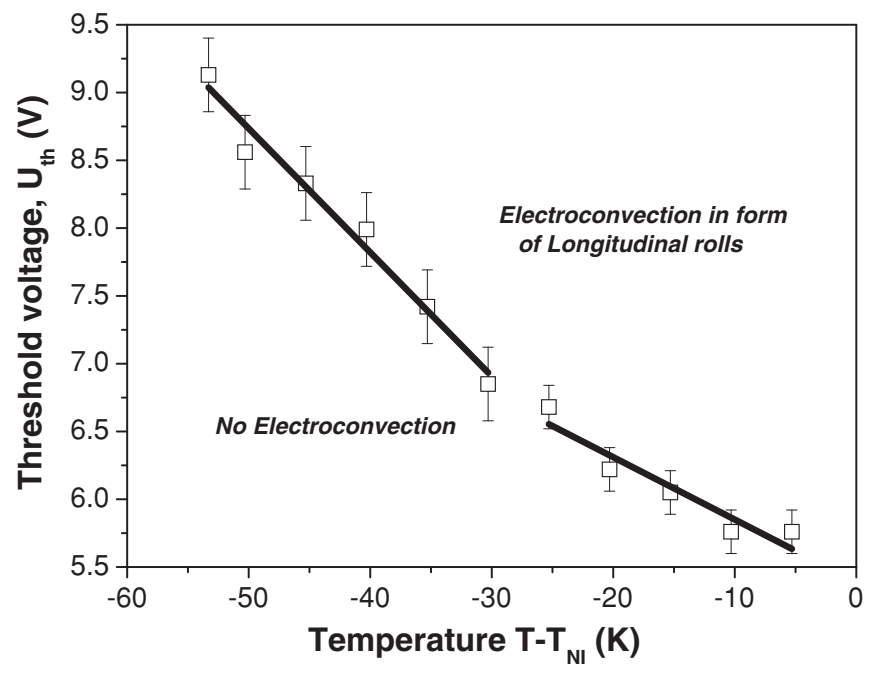

FIG. 7. Threshold voltage as a function of reduced temperature $\left(T-T_{\mathrm{NI}}\right)$ for the observation of ns-EC in form of longitudinal rolls at a frequency of $1 \mathrm{kHz}$. Two separate linear fits distinguish the regimes that have been reported as uniaxial and biaxial phases.

\section{TEMPERATURE DEPENDENCE OF THE THRESHOLD VOLTAGE}

As seen in Sec. IV above, longitudinal rolls were observed at frequencies above $\sim 100 \mathrm{~Hz}$ up to $\sim 1 \mathrm{kHz}$, depending on the magnitude of the applied voltage. The threshold voltage of the LRs is also temperature dependent; Fig. 7 shows the threshold voltage (at $1 \mathrm{kHz}$ ) for the appearance of EC (in the form of longitudinal rolls) as a function of the reduced temperature, $T-T_{\mathrm{NI}}$ where $T_{\mathrm{NI}}$ is the nematic to isotropic phase transition temperature. As the temperature decreases, the threshold voltage increases. Two distinct regimes can be observed in Fig. 7, both of which have a linear dependence of threshold voltage on temperature. The gradients are $-0.046 \pm$ $0.009 \mathrm{~V} / \mathrm{K}$ and $-0.091 \pm 0.007 \mathrm{~V} / \mathrm{K}$ in the high and low temperature regions, respectively. The temperature at which the behavior changes is around $T-T_{\mathrm{NI}}=-30 \mathrm{~K}$, the same temperature as has been reported as the $N_{u}$ to $N_{b}$ transition in this bent-core material [31].

The threshold voltage was also determined as a function of frequency, Fig. 8, which shows $U_{\text {th }}$ as a function of frequency in a $5.1 \mu \mathrm{m}$ thick sample at three different temperatures $\left(T-T_{\mathrm{NI}}=-7 \mathrm{~K},-27 \mathrm{~K}\right.$, and $\left.-47 \mathrm{~K}\right)$. It can be seen that $U_{\text {th }}$ increases linearly with frequency and that the steepness of $U_{\mathrm{th}}(f)$ increases slightly with decreasing temperature. A similar, linear dependence of the threshold voltage of ns-EC with frequency has also been observed by other researchers [13-15].

\section{CHARACTERISTICS OF THE EC PATTERN ACROSS THE NEMATIC PHASE}

The previous sections detailed the various pattern formations in the bent-core material with changing frequency and temperature. This section presents further details that distinguish the EC in the high and low temperature regions of the nematic phase. Figure 9 shows EC in the form of LR at $1.5 U_{\text {th }} \pm 0.2 \mathrm{~V}$ and at a frequency of $1 \mathrm{kHz}$ at different

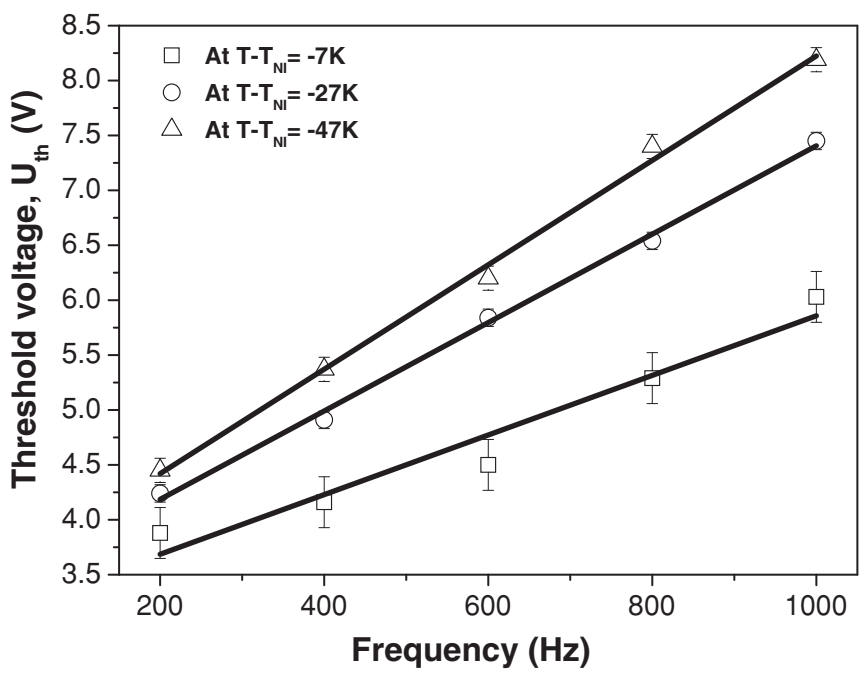

FIG. 8. Frequency dependence of the threshold voltage of longitudinal rolls at three different reduced temperatures $T-T_{\mathrm{NI}}=-7$, $-27,-47 \mathrm{~K}$ covering nearly the complete nematic regime. The solid lines represent linear fits to the data.

temperatures. In the high temperature regime, a clear display of LR is seen [Fig. 9(a), $5 \mathrm{~K}$ below $T_{\mathrm{NI}}$ ] which is typical of patterns obtained until $T-T_{\mathrm{NI}}=-30 \mathrm{~K}$ [Figs. 9(b) and 9(c)]. At lower temperatures, one does not observe a clear display of LRs; instead an obscure view is observed as seen in Figs. 9(d)-9(f), which are at $T-T_{\mathrm{NI}}=-35 \mathrm{~K},-50 \mathrm{~K}$, and $-55 \mathrm{~K}$, respectively. It is difficult to define the temperature threshold for the "haze" that appears to cover the LR at lower temperatures, but the two unique regimes clearly exist.

A quantitative measure of the critical wave vector of the rolls (LRs) has been determined by deducing the wavelength $\lambda$, of the rolls. The dimensionless wave number $q_{c}$ is defined as $q_{c}=2 d / \lambda$ where $d$ is thickness of the sample cell, 5.1 $\mu \mathrm{m}$ in the present case. Figure 10 shows $q_{c}$ as a function of reduced temperature at a frequency of $1 \mathrm{kHz}$ and voltage $1.5 U_{\text {th }} \pm 0.2 \mathrm{~V}$ (it is not possible to keep the voltage constant for these measurements as the EC patterns are voltage dependent, hence the choice of $1.5 U_{\mathrm{th}}$ ). As can be seen from Fig. 10, $q_{c}$ shows no significant temperature dependence at lower temperatures, while at higher temperatures, it strongly decreases with increasing temperature. The roll wavelength changes from $11.40 \pm 0.05 \mu \mathrm{m}$ at $T-T_{\mathrm{NI}}=-5 \mathrm{~K}$ to $7.70 \pm$ $0.05 \mu \mathrm{m}$ at $T-T_{\mathrm{NI}}=-30 \mathrm{~K}$ and thereafter varies between $7.40 \pm 0.05 \mu \mathrm{m}$ and $6.95 \pm 0.05 \mu \mathrm{m}$. This again indicates two distinct regimes in the nematic phase.

Apart from the oblique and longitudinal rolls, the other instability observed is the skewed varicose or bimodal varicose, occurring at voltages much higher than the threshold for EC. This instability also shows an interesting behavior with respect to the high and low temperatures in the nematic phase. At $T-T_{\mathrm{NI}}=-13 \mathrm{~K}$, applying a voltage of $>16 V_{\mathrm{rms}}$ causes a transition from a traveling LR to a crisscrossed EC configuration. Figure 11(a) shows the textural image at $900 \mathrm{~Hz}, 1.25 U_{\text {th }}^{\mathrm{cc}}$ where $U_{\mathrm{th}}^{\mathrm{cc}}=16 V_{\mathrm{rms}}$ is the threshold voltage for the crisscrossed EC. At this reduced temperature, the crisscrossed EC is formed at threshold frequencies starting at $600 \mathrm{~Hz}$ at $16 \mathrm{~V}$ and increasing to $900 \mathrm{~Hz}$ at $20 \mathrm{~V}$. On 

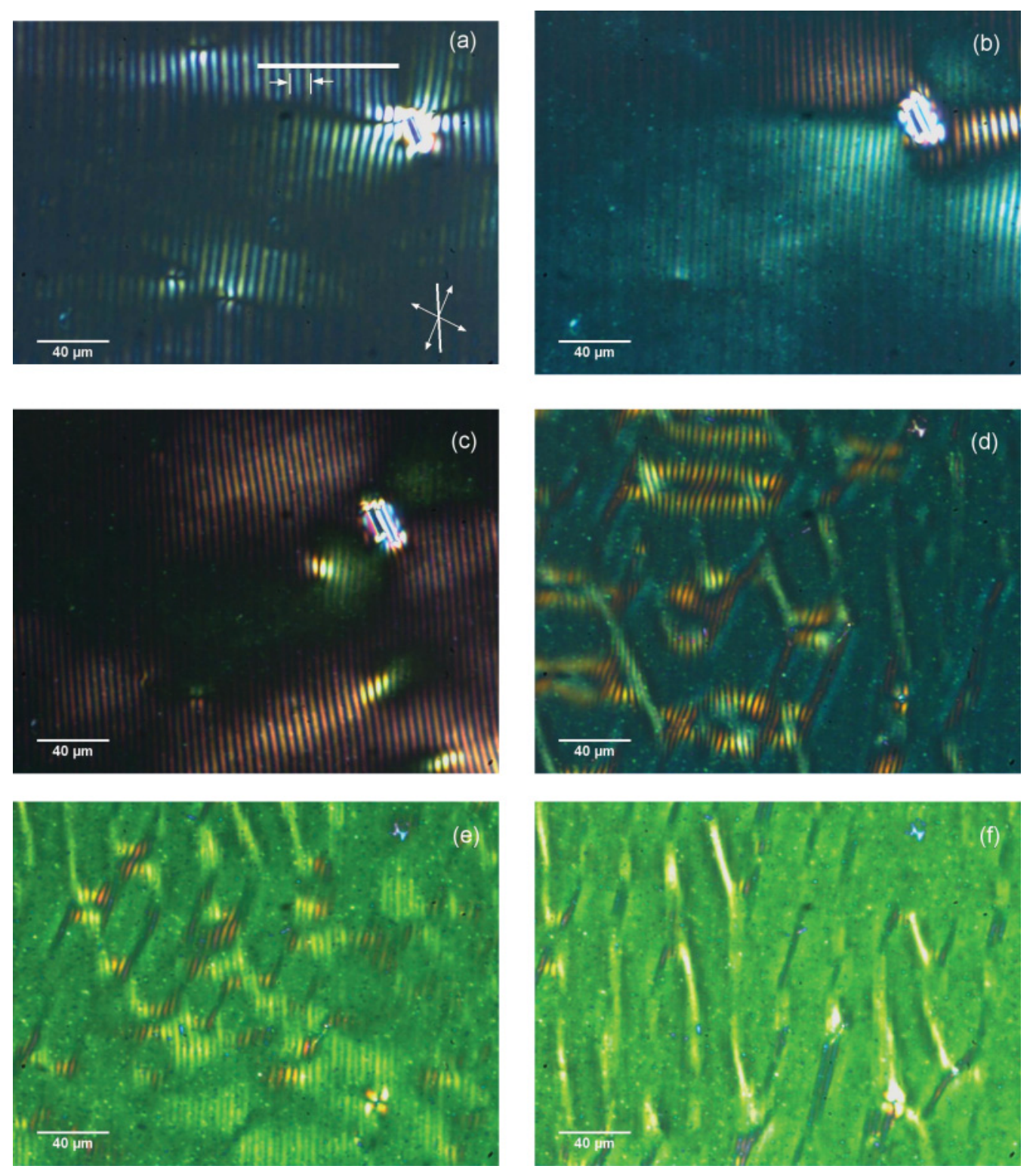

FIG. 9. (Color online) EC patterns in form of longitudinal rolls observed $1 \mathrm{kHz}$ and approximately $1.5 U_{\text {th }} \pm 0.2 \mathrm{~V}$ at reduced temperatures of (a) $-5 \mathrm{~K}$, (b) $-15 \mathrm{~K}$, (c) $-25 \mathrm{~K}$, (d) $-35 \mathrm{~K}$, (e) $-50 \mathrm{~K}$, (f) $-55 \mathrm{~K}$. The director is along the roll direction, i.e., in the almost vertical direction as marked by the solid line in the bottom right hand corner of (a). The double-headed arrows show the orientation of the crossed polarizers. The scale is in the lower left hand corner in each picture. The horizontal white line in (a) was used to measure the intensity profile for $9.7 V_{\text {rms }}$ as shown in Fig. 12. The wavelength $\lambda$ of the longitudinal rolls is shown by the distance between the arrows in (a).

decreasing the temperature further, one does not observe much change in the development of the criss-crossed EC pattern until $T-T_{\mathrm{NI}}=-33 \mathrm{~K}$; see Fig. 11(b), which shows the pattern at $320 \mathrm{~Hz}, 1.15 U_{\mathrm{th}}^{\mathrm{cc}}$ where $U_{\mathrm{th}}^{\mathrm{cc}}=13 V_{\mathrm{rms}}$. As the temperature is decreased further, the pattern is observed at lower frequencies and lower threshold voltages. At $T-T_{\mathrm{NI}}=$ $-38 \mathrm{~K}$, instead of the direct transition from traveling LR to crisscrossed pattern, the EC follows an indirect route via skewed varicose or bimodal varicose instabilities at low frequencies and lower threshold voltages. Figure 11(c) shows the skewed varicose or bimodal varicose patterns at a frequency of $180 \mathrm{~Hz}, 1.14 U_{\mathrm{th}}^{\mathrm{bv}}$ where $U_{\mathrm{th}}^{\mathrm{bv}}=14 V_{\mathrm{rms}}$ is the threshold voltage for the appearance of skewed varicose or bimodal varicose EC. This pattern of EC evolves gradually with changing temperature and is not observed at temperatures above $T-T_{\mathrm{NI}}=-38 \mathrm{~K}$. The sequence of patterns observed at $T-T_{\mathrm{NI}}=-38 \mathrm{~K}$ is "traveling OR and traveling LR"-"two rolls of different orientation"-"skewed varicose or bimodal varicose"-"crisscrossed pattern." Figure 11(d) shows the two rolls of different orientation beginning to form the skewed varicose or bimodal varicose pattern, seen in a fully developed form in Fig. 11(c). This pattern can be identified as a bimodal varicose instability because it indicates the transition to a bimodal state, composed of the superposition of two roll systems with different orientation [33]. The angle (marked in the figure with the bold yellow lines) between the two orientations is $115 \pm 2^{\circ}$. The bold white line is the rubbing direction along which at lower frequencies the LR pattern 


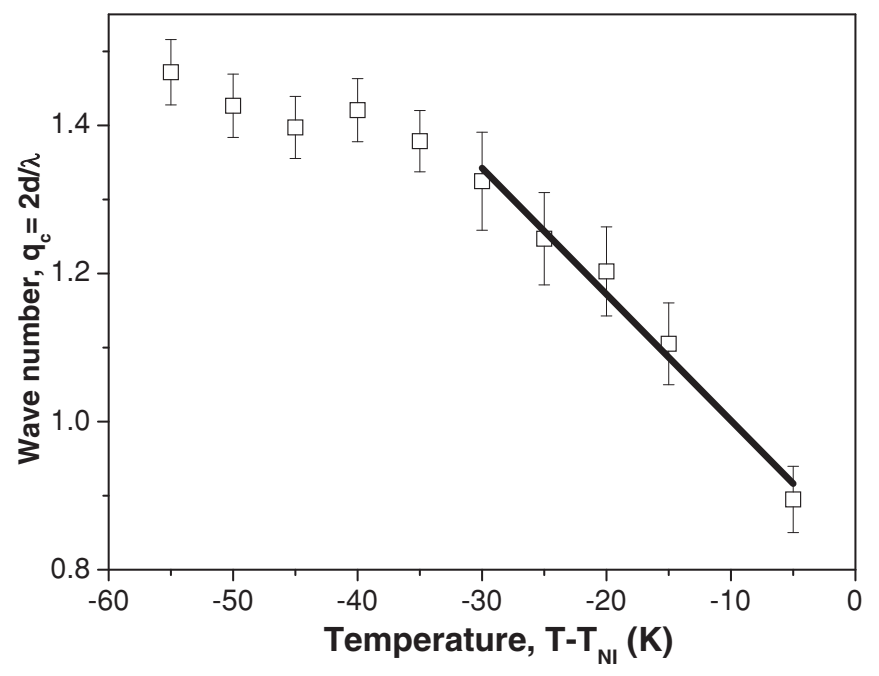

FIG. 10. Wave number $q_{c}(=2 d / \lambda)$ of the longitudinal rolls as a function of reduced temperature at $1 \mathrm{kHz}$ and approximately $1.5 U_{\mathrm{th}} \pm$ $0.2 \mathrm{~V}$.

was observed. The intersection of the two stripes forms a closed grid with one of the axes along the rubbing direction $\left( \pm 5.0-7.0^{\circ}\right)$ with typical dimensions of $5.30 \pm 0.05 \mu \mathrm{m}$ length and $3.00 \pm 0.05 \mu \mathrm{m}$ width. The bimodal varicose instability is observed in the low end temperature regime near the Sm- $X$ phase, at low frequencies between 120 and $500 \mathrm{~Hz}$, and at $\sim 14 V_{\text {rms }}$ and above. The high temperature nematic regime does not exhibit this bimodal instability.

Another point of dissimilarity in the patterns observed in the high and low end temperatures is the observance of moving diagonal stripes in the nematic region near the $\mathrm{Sm}-X$ phase. Figure 11(e) shows the emergence of diagonal stripes at $T-$ $T_{\mathrm{NI}}=-50 \mathrm{~K}$, at a frequency of $80 \mathrm{~Hz}$ and voltage of $16 V_{\mathrm{rms}}$. The origin and the angle of stripes are quite different from the stripes forming the bimodal pattern at higher frequencies. The acute angle formed between the stripes and the rubbing direction is $62 \pm 1^{\circ}$ [shown by the bold white stripe in Fig. 11(e)] whereas the angle formed by the bimodal instability at higher frequency relative to the rubbing direction is $30 \pm 1^{\circ}$ [shown by the bold white stripe in Fig. 11(d)]. Hence these diagonal stripes do not seem to be related to the bimodal pattern formed and represent another type of EC at low frequencies. Although further work is required to explain these diagonal patterns in detail; their appearance is worth mentioning here as yet another difference exhibited in the EC behavior of this material in the high and low temperature regimes, reported as uniaxial and biaxial nematic regions, respectively.

\section{SUMMARY}

This paper highlights the study of electroconvection in a bent-core oxadiazole material, revealing that the system exhibits nonstandard-electroconvection across the entire nematic regime, mainly in the form of longitudinal rolls. Other forms of EC, including oblique rolls, bimodal varicose, and turbulence, are also observed. The first part of the paper described the different types of rolls that were observed at a specific temperature, elucidating what is typical for the system. In particular, the characteristics of roll wavelength as a function of both frequency and voltage were described and contrasted with observations made by others revealing the distinct properties of this material. The ns-EC patterns observed cannot be described by the Carr-Helfrich model and the basic distinguishing features are as follows:

(i) Near the onset, the patterns cannot be observed by the shadowgraph method, and crossed or nearly crossed polarizers are needed to observe them.

(ii) The contrast of the pattern is very weak at the onset (compared to standard EC patterns). This is clearly illustrated by Fig. 12, which shows the intensity profile [measured along the white line shown in Fig. 9(a)] as a function of displacement at $T-T_{\mathrm{NI}}=-13 \mathrm{~K}$ for different applied voltages.

(iii) The behavior of threshold voltage $U_{\text {th }}$ with respect to frequency is linear (Fig. 8) whereas in standard EC an increase in threshold voltage with increasing frequency has been reported [13-15].

It is also worth commenting on whether the rolls we observe should be termed "longitudinal" or "parallel," to allow a full comparison with the literature. Wiant et al. reported parallel rolls of period less than $d$ in a $(-,-)$ material over a small frequency region $(1-28 \mathrm{~Hz})$ [14]. Toth-Katona et al. observed longitudinal rolls oriented along the rubbing direction with period less than $d$ in a $(-,-)$ material over a range of few hundred $\mathrm{Hz}$ [15]. Heuer et al. also called the stripes of the order of cell gap "longitudinal rolls" for a $(+,-)$ material [10]. Tadapatri et al. distinguished between parallel and longitudinal rolls using the following criteria:

(i) PRs occur only in $(-,-)$ materials over a small frequency range of $1-28 \mathrm{~Hz}$ whereas LRs can occur in $(-,-)$ and $(-,+)$ regions.

(ii) The period of PRs is less than $d$ whereas it is $2 d$ and $3 d$ for LRs.

(iii) The threshold voltage for PRs is linear in frequency whereas for the LR state, they report three broadly linear parts with different slopes.

(iv) PRs are localized and LRs are extended [16].

For this oxadiazole material, the rolls occur over a broad frequency range and the wavelength of the rolls varies between $d$ and $2 d$, depending on the frequency and voltage of the applied field. The rolls are observed only in the $(-,+)$ regime [not the high frequency $(-,-)$ region], the period of rolls is $2 d$, and the threshold is linear in frequency. Moreover, the rolls observed by us make an angle of 5.0-7.0 $0^{\circ}$ with the rubbing direction. Combining all the features of the rolls we observe, we consider that they should be termed longitudinal and not parallel rolls, in agreement with Tadapatri et al. [16].

In common with our observations, Wiant et al. also report a linear behavior of the temperature dependence of the threshold voltage $U_{\text {th }}$ of parallel stripes in a bent-core nematic material [14]. The (uniaxial) nematic regime in their case was $18 \pm 1 \mathrm{~K}$ wide and the linear behavior was reported over an $8 \mathrm{~K}$ temperature range near the clearing point. Tanaka et al. [34] also report the behavior of ns-EC with temperature in the nematic regime of a binary mixture of the compound in [14] and a rod-like nematic material. Comparing the various phenomena, the nonlinear dependence of threshold voltage with temperature [Fig. 8(a) of Ref. [34]] is a characteristic of the prewavy regime, unlike the longitudinal rolls observed in our case. Further, the temperature range studied in [34] is $18 \mathrm{~K}$ 

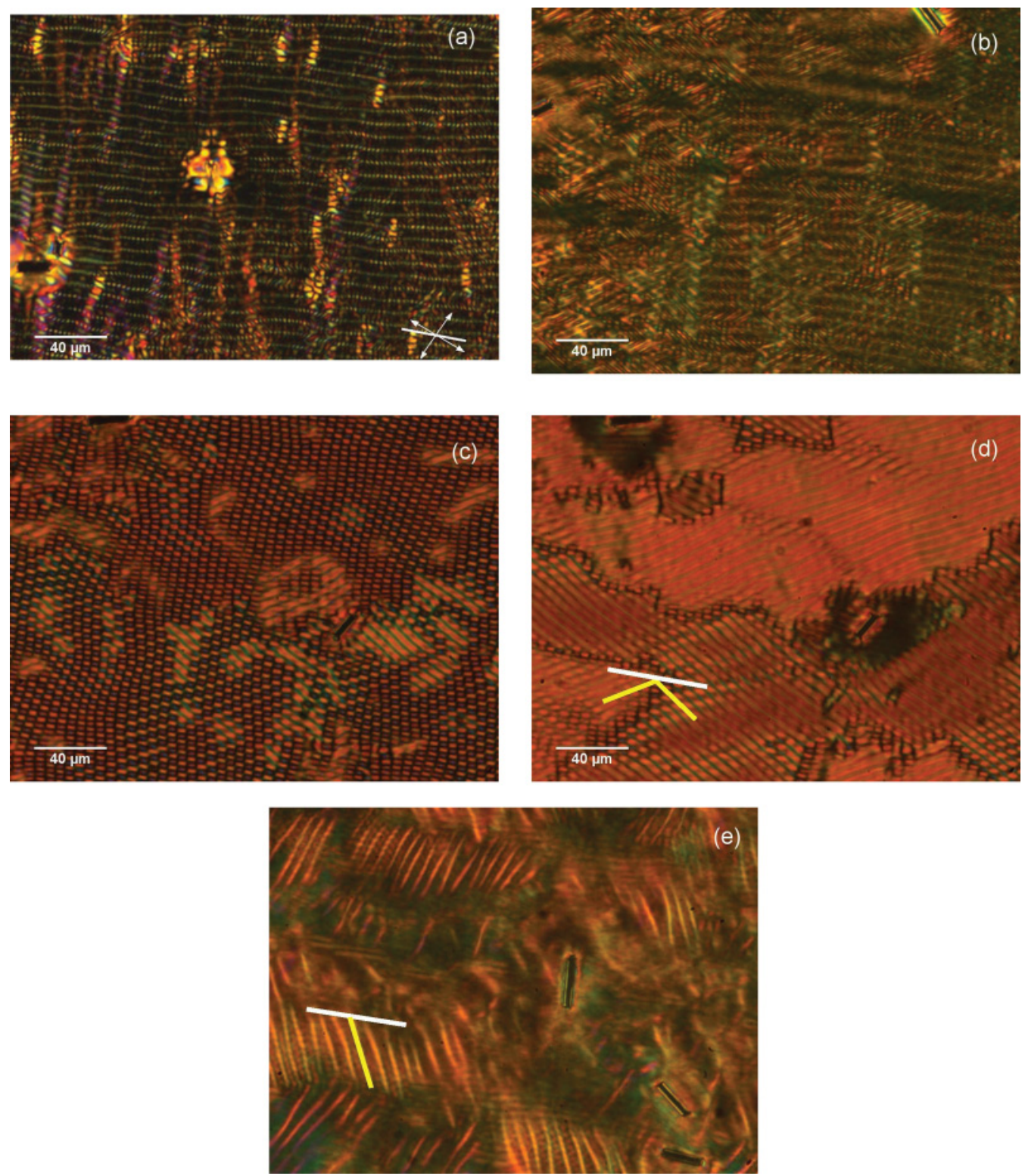

FIG. 11. (Color online) Instabilities observed much above the threshold of longitudinal rolls. Crisscrossed pattern at (a) $T-T_{\mathrm{NI}}=-13 \mathrm{~K}$, $900 \mathrm{~Hz}, 1.25 U_{\mathrm{th}}^{\mathrm{cc}}$ where $U_{\mathrm{th}}^{\mathrm{cc}}=16 V_{\mathrm{rms}}$, and (b) $T-T_{\mathrm{NI}}=-33 \mathrm{~K}, 320 \mathrm{~Hz}, 1.15 U_{\mathrm{th}}^{\mathrm{cc}}$ where $U_{\mathrm{th}}^{\mathrm{cc}}=13 V_{\mathrm{rms}}$. Bimodal pattern at $(\mathrm{c}) T-T_{\mathrm{NI}}=$ $-38 \mathrm{~K}, 180 \mathrm{~Hz}, 1.14 U_{\mathrm{th}}^{\mathrm{bv}}$ where $U_{\mathrm{th}}^{\mathrm{bv}}=14 V_{\mathrm{rms}}$. Two rolls of different orientation forming bimodal pattern at (d) $T-T_{\mathrm{NI}}=-38 \mathrm{~K}, 140 \mathrm{~Hz}$, $1.14 U_{\text {th }}^{\text {bv }}$ where $U_{\text {th }}^{\text {bv }}=14 V_{\text {rms. }}$ (e) Diagonal stripes (with an angle different to the angle of the bimodal rolls in (d)) $T-T_{\mathrm{NI}}=-50 \mathrm{~K}, 80 \mathrm{~Hz}$, $V=16 V_{\text {rms }}$. The rubbing direction is almost horizontal as marked in the bottom right hand corner of (a). The double-headed arrows show the orientation of the crossed polarizers. The scale is in the lower left hand corner in each picture.

which is much narrower than the $55 \mathrm{~K}$ range reported here, and the frequency was much higher $(200 \mathrm{kHz})$ than for our oxadiazole system $(1 \mathrm{kHz})$.

As mentioned earlier, one cannot utilize the Carr-Helfrich model to explain the observation of the patterns obtained in the oxadiazole system. Some authors have interpreted the formation of longitudinal and parallel rolls of the order of $d$ at low [18-20] and high [19] frequency via the flexoelectric effect. Madhusudana et al. [20] proposed the inclusion of a flexoelectric term in the Carr-Helfrich model to account for the LR patterns reported in [19] at high frequency. As mentioned in Sec. IV, flow patterns are a feature of the EC observed in our material and Tóth-Katona et al., who also observed LR with flow, proposed the inclusion of flexoelectricity in the standard model to account for the behavior [15]. The flexoelectric coefficients of this material have not yet been determined, but it may be important to include such terms in a full explanation of the observed EC pattern. Finally, we note that it is possible that the electrodiffusion model of Treiber et al. [35] could also explain the nonstationary waves as observed.

Our observance of ns-EC in a regime in which $\Delta \varepsilon<0$ and $\Delta \sigma>0$ might indicate that the sign of the conductivity anisotropy is not a decisive factor for the formation of the LR, in agreement with the suggestion of Tadapatri et al. [16]. If conductivity is, say, one of the absolute factors then one might think of a pre-inversion effect caused by the changing sign of 


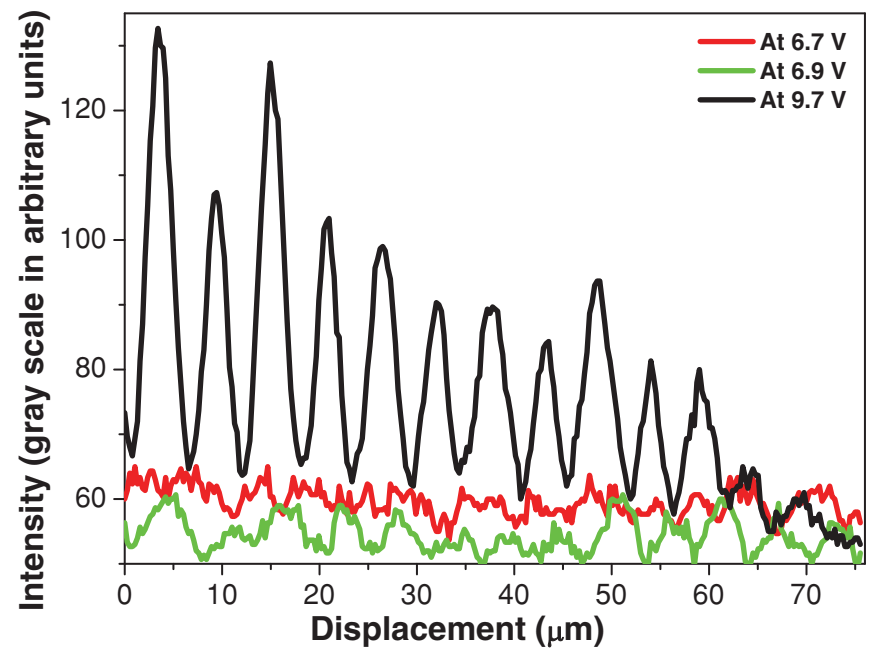

FIG. 12. (Color online) Intensity profile (gray scale) along the bold horizontal line (perpendicular to the longitudinal rolls) marked in Fig. 9(a) for 9.7 $V_{\text {rms }}$ (black line), 6.9 $V_{\text {rms }}$ (green line: faint gray scale), and $6.7 V_{\text {rms }}$ (red line: intermediate gray scale) as a function of displacement. The frequency used is $1 \mathrm{kHz}$ and the temperature is $T-T_{\mathrm{NI}}=-13 \mathrm{~K}$. The low contrast and inhomogeneity of the rolls is apparent at the onset, becoming much improved at higher voltages.
$\Delta \sigma$ [as seen in the inset to Fig. 3(c)], resulting in the ns-EC. We agree with Tadapatri et al. that in the present understanding, any theory developed to account for the anisotropic convection in bent-core systems, cannot ignore the complex electrical parameters [16].

One of the significant findings for our system is the distinct behavior of $\mathrm{EC}$ in the high and low temperature regimes of the material (Figs. 9-11). A continuous texture change occurs across the regime reported as a biaxial to uniaxial nematic phase transition [31]. Specifically, the wave number $q_{c}$ representing the roll wavelength is almost constant in the biaxial regime, but strongly temperature dependent in the uniaxial regime. Also, at lower temperatures, the EC follows an indirect route from LRs to a crisscrossed pattern, via a bimodal varicose instability (Fig. 11). The EC measurements clearly show that two different temperature regimes occur in this nematic system, with transitions in behavior at the same temperature as the reported uniaxial to biaxial transition.

\section{ACKNOWLEDGMENTS}

This work was supported by the Engineering and Physical Sciences Research Council under Project No. EP/G023093/1. The authors acknowledge valuable discussions with Ying Xiang.
[1] L. Kramer and W. Pesch, Annu. Rev. Fluid Mech. 27, 515 (1995).

[2] A. Buka, N. Éber, W. Pesch, and L. Kramer, in Self-Assembly, Pattern Formation and Growth Phenomena in Nano-Systems, edited by A. A. Golovin and A. A. Nepomnyashchy (Springer, Secausus, NJ, 2006), p. 55.

[3] L. Kramer and W. Pesch, in Physical Properties of Nematic Liquid Crystals, edited by D. A. Dunmur, A. Fukuda, and G. R. Luckhurst (Inspec, London, 2001), p. 441.

[4] W. Pesch and L. Kramer, in Pattern Formation in Liquid Crystals, edited by A. Buka and L. Kramer (Springer, New York, 1996), p. 69.

[5] E. F. Carr, Mol. Cryst. Liq. Cryst. 7, 253 (1969).

[6] W. Helfrich, J. Chem. Phys. 51, 4092 (1969).

[7] E. Bodenschatz, W. Zimmermann, and L. Kramer, J. Phys. (Paris) 49, 1875 (1988).

[8] Á. Buka, B. Dressel, W. Otowski, K. Camara, T. Toth-Katona, L. Kramer, J. Lindau, G. Pelzl, and W. Pesch, Phys. Rev. E 66, 051713 (2002).

[9] R. Stannarius and J. Heuer, Eur. Phys. J. E 24, 27 (2007).

[10] J. Heuer, R. Stannarius, M.-G. Tamba, and W. Weissflog, Phys. Rev. E 77, 056206 (2008).

[11] B. Dressel and W. Pesch, Phys. Rev. E 67, 031707 (2003).

[12] P. Kumar, U. S. Hiremath, C. V. Yelamaggad, A. G. Rossberg, and K. S. Krishnamurthy, J. Phys. Chem. B 112, 9753 (2008).

[13] E. Kochowska, S. Németh, G. Pelzl, and Á. Buka, Phys. Rev. E 70, 011711 (2004).

[14] D. Wiant, J. T. Gleeson, N. Éber, K. Fodor-Csorba, A. Jákli, and T. Tóth-Katona, Phys. Rev. E 72, 041712 (2005).
[15] T. Tóth-Katona, A. Cauquil-Vergnes, N. Éber, and Á. Buka, Phys. Rev. E 75, 066210 (2007).

[16] P. Tadapatri, U. S. Hiremath, C. V. Yelamaggad, and K. S. Krishnamurthy, J. Phys. Chem. B 114, 10 (2010).

[17] P. Tadapatri, U. S. Hiremath, C. V. Yelamaggad, and K. S. Krishnamurthy, J. Phys. Chem. B 114, 1745 (2010).

[18] M. Goscianski and L. Leger, J. Phys. Colloques 36, C1-231 (1975).

[19] L. M. Blinov, M. I. Barnik, V. T. Lazareva and A. N. Trufanov, J. Phys. Colloques 40, C3-263 (1979).

[20] N. V. Madhusudana and V. A. Raghunathan, Liq. Cryst. 5, 1789 (1989).

[21] A. Krekhov, W. Pesch, N. Éber, T. Tóth-Katona, and Á. Buka, Phys. Rev. E 77, 021705 (2008).

[22] Á. Buka, N. Éber, W. Pesch, and L. Kramer, Phys. Rep. 448, 115 (2007).

[23] S. Kai and K. Hirakawa, Solid State Commun. 18, 1573 (1976).

[24] J.-H. Huh, Y. Hidaka, Y. Yusuf, N. Eber, T. Toth-Katona, Á. Buka, and S. Kai, Mol. Cryst. Liq. Cryst. 364, 111 (2001).

[25] A. N. Trufanov, L. M. Blinov, and M. I. Barnik, Sov. Phys. JETP 51, 314 (1980).

[26] H. Takezoe and Y. Takanishi, Jpn. J. Appl. Phys. 45, 597 (2006).

[27] G. R. Luckhurst, Thin Solid Films 393, 40 (2001).

[28] L. A. Madsen, T. J. Dingemans, M. Nakata, and E. T. Samulski, Phys. Rev. Lett. 92, 145505 (2004); B. R. Acharya, A. Primak, T. J. Dingemans, E. T. Samulski, and S. Kumar, Pramana 61, 231 (2003); B. R. Acharya, A. Primak, and S. Kumar, Phys. Rev. Lett. 92, 145506 (2004). 
[29] O. Francescangeli, F. Vita, C. Ferrero, T. Dingemans, and E. T. Samulski, Soft Matter 7, 895 (2011).

[30] C. D. Southern, P. D. Brimicombe, S. D. Siemianowski, S. Jaradat, N. Roberts, V. Görtz, J. W. Goodby, and H. F. Gleeson, Euro. Phys. Lett. 82, 56001 (2008).

[31] Y. Xiang, J. W. Goodby, V. Görtz, and H. F. Gleeson, Appl. Phys. Lett. 94, 193507 (2009).
[32] P. A. Penz, Phys. Rev. Lett. 24, 1405 (1970)

[33] L. Kramer, B. Dressel, H. Zhao, and W. Pesch, Mol. Cryst. Liq. Cryst. 364, 101 (2001).

[34] S. Tanaka, H. Takezoe, N. Eber, K. Fodor-Csorba, A. Vajda, and A. Buka, Phys. Rev. E 80, 021702 (2009).

[35] M. Treiber and L. Kramer, Mol. Cryst. Liq. Cryst. 261, 311 (1995). 